\title{
Barreras psicológicas y psicosociales a la mediación en la resolución de conflictos en contextos universitarios
}

\author{
Carlos María Alcover \\ Exdefensor de la Universidad Rey Juan Carlos
}

\section{Resumen}

La utilización de la mediación como una estrategia prioritaria, aunque no exclusiva, para la gestión y la resolución de conflictos en un número creciente de contextos tiende a generar expectativas elevadas acerca de su potencial eficacia. Sin embargo, a pesar de su indudable éxito en la solución de determinados tipos de conflictos, no siempre permite el logro de los resultados esperados. Identificar y comprender los factores que dificultan la consecución de acuerdos y reducen la eficacia de la mediación representa un objetivo fundamental, tanto para el intento de superar estas barreras como para evitar la frustración causada por el incumplimiento de las expectativas vinculadas a ella. Los objetivos de este artículo, dirigido especialmente a quienes desempeñan el cargo de Defensores Universitarios, se centran en primer lugar, en sintetizar las claves, los objetivos y los beneficios de la mediación en el ámbito universitario, y en segundo lugar, en el análisis de las principales barreras psicológicas y psicosociales a la mediación.

\section{Mediación y seguridad psicológica}

En un artículo anterior publicado en esta misma revista (Alcover, 2016), se señaló que la utilización de la mediación en la resolución de conflictos en contextos universitarios por los Defensores y las Defensoras Universitarios había ampliado las competencias atribuidas habitualmente a las figuras de ombudsperson, lo que les permitía intervenir como agentes activos implicados en la búsqueda de soluciones que no solo contribuyen a resolver el conflicto, sino también a restablecer, restaurar o mejorar las relaciones entre las partes. La mediación consiste en la intervención en un conflicto 
de un tercero neutral, con el propósito de ayudar a las partes a resolver sus problemas en un ambiente psicológico seguro. La mediación es una negociación dirigida por una persona experta en facilitar la comunicación y el entendimiento entre las partes (Moore, 2014). Puede tener un carácter formal -cuando existe un marco regulador del procedimiento y una definición del alcance del proceso- o informal -en el caso de que se trate de un procedimiento utilizado de manera flexible y definido por las partes y el/la mediador/a.

En contextos universitarios, la mediación puede utilizarse en conflictos horizontales, es decir, cuando el conflicto tiene como actores a personas o grupos que pertenecen al mismo sector y cuentan con niveles de poder similares, y en conflictos verticales, esto es, cuando el conflicto tiene como actores a personas o grupos que pertenecen a sectores diferentes y existe una asimetría de poder entre ellas (Alcover, 2009). La mediación resulta más indicada en ciertos tipos de conflictos, en los cuales se comprueba que es más frecuente su utilización y suele ser mayor su eficacia (Alcover, 2011):

- Conflictos de intereses o metas incompatibles.

- Escasez de recursos.

- Problemas de relaciones interpersonales.

- Conflictos de poder (real o simbólico).

- Percepciones e interpretaciones acerca de normativas, toma de decisiones, trato recibido, etc.

- Problemas de comunicación y de información.

Las claves de la mediación pueden sintetizarse en las siguientes:

- Voluntariedad de las partes en iniciar el proceso de mediación.

- Expresión de un interés real y una implicación en resolver la situación a través de la comunicación abierta, el diálogo y al búsqueda conjunta de soluciones que conduzcan a un acuerdo.

- Aceptación explícita del/la mediador/a como alguien neutral e independiente que va a facilitar el proceso.

- Respeto mutuo entre las partes.

- Equilibrio de poder entre las partes en el contexto de la mediación.

- Libertad de abandonar el proceso cuando se desee.

- Seguridad psicológica de las partes.

Todos estos elementos son clave para crear las condiciones que faciliten la eficacia de la mediación, aunque por sí solas no puedan garantizar el éxito en todos los casos, pero resulta especialmente interesante el último concepto mencionado, relativo a la seguridad psicológica. Se trata de un con- 
cepto desarrollado inicialmente por Amy Edmonson (1999), profesora de la Universidad de Harvard, para referirse a un elemento fundamental para la eficacia del trabajo en equipo, pero que puede aplicarse perfectamente al contexto de cualquier relación interpersonal, como ocurre en un proceso de mediación. La seguridad psicológica se define como "un clima de equipo que se caracteriza por la confianza interpersonal y el respeto mutuo en el que las personas se sienten cómodas las unas con las otras". Aplicado a un contexto de grupo, se trata de "la creencia compartida por parte de los miembros de un grupo de que el contexto grupal es seguro a la hora de la asunción de riesgos interpersonales", en el sentido de expresar opiniones, realizar aportaciones, proponer sugerencias o realizar críticas. La seguridad psicológica sería entonces "una sensación de confianza en que el equipo no hará sentir vergüenza, no rechazará y no castigará a nadie por decir en alto lo que piensa" (Edmondson, 1999, p. 354). Si se extrapola el concepto al contexto de una mediación, la seguridad psicológica se refiere a la sensación de las partes implicadas en ella de que serán tratadas con respeto, de que podrán expresar sus sentimientos, pensamientos y opiniones sin temor a ser censuradas, criticadas o menospreciadas, y de sentir la confianza y la seguridad de que no sufrirán críticas o ataques personales. En este sentido, el papel del/la mediador/a es fundamental para construir el clima de seguridad psicológica en la mediación, y lograr que el comportamiento de las partes permita la percepción mutua de tal clima.

La mediación es una estrategia de resolución de conflictos basada en la denominada justicia relacional, que se refiere a formas de justicia de abajo hacia arriba, que se producen como resultado de la conducta cooperativa, el acuerdo, la negociación y el diálogo directo entre las partes cuando se reconoce la existencia de un conflicto (Casanovas y Poblet, 2008). Además de la mediación, otras modalidades de resolución de conflictos basadas en la justicia relacional son la justicia restaurativa, la mediación ofensor-víctima, la justicia transicional y la justicia comunitaria.

En definitiva, la mediación se basa en la clara voluntad de las partes en lograr un acuerdo a través del diálogo directo y la búsqueda de soluciones conjunta, en la aceptación de centrarse en los intereses de ambas, abandonando la apelación a las posiciones (el poder) y a los derechos (lo que me corresponde), y en la implicación responsable en formular propuestas y ceder conjuntamente lo necesario para lograr acuerdos que satisfagan de forma equilibrada los intereses de ambas.

Como ya se mencionó al comienzo, es importante que tanto el/la mediador/a como las partes participantes en el proceso se impliquen en la búsqueda de soluciones que no solo contribuyan a resolver el conflicto, sino también a restablecer, restaurar o mejorar las relaciones entre las partes. En consecuencia, la necesidad de adecuar la estrategia de mediación a 
utilizar con el ánimo de lograr este doble objetivo ha dado lugar al concepto de eficacia diádica (Bush y Folger, 1994; Kressel y Pruitt,1989): la mediación ha de servir para encontrar una solución o un acuerdo que satisfaga en la mayor medida posible los intereses de las partes, y simultáneamente ha de procurar que permita la redefinición de las relaciones entre ellas, facilitando el mantenimiento de la relación a lo largo del tiempo. Teniendo en cuenta las características de los contextos universitarios, donde las relaciones entre los miembros de la comunidad universitaria tienden a ser duraderas o a prolongarse durante periodos de tiempo significativos, este segundo objetivo de la mediación resulta de gran relevancia (Alcover, 2009). En este sentido, el empleo durante el proceso de mediación de estrategias que faciliten su logro puede incrementar la eficacia de las intervenciones realizadas por los defensores y las defensoras en conflictos universitarios.

\section{Principales barreras psicológicas y psicosociales a la mediación}

Los resultados de la investigación y la experiencia acumulada de la práctica profesional en diferentes contextos donde es de aplicación frecuente han permitido identificar una serie de beneficios de la mediación (Alcover, 2009, 2012; Bush y Folger, 1994; Moore, 2014; Poitras, 2009):

- Tanto en la conciliación como en la mediación siguen siendo las partes las que tienen la capacidad de tomar las decisiones por sí mismas y llegar a un acuerdo.

- Se ha comprobado que los mecanismos más eficaces (es decir, los que permiten alcanzar las mejores soluciones y las más duraderas en su cumplimiento) son aquellos en los que las partes llegan por sí mismas a un acuerdo.

- La mediación provee las bases para que las partes implicadas se sientan protagonistas tanto del proceso seguido como de los resultados obtenidos.

- Aumenta su nivel de participación en los procesos de toma de decisiones que conducen a acuerdos, e incrementa sus niveles de empoderamiento.

- Preserva el concepto de libertad y autonomía académicas, ya que es un procedimiento interno de resolución de conflictos.

- Permite a los miembros de la comunidad universitaria implicarse en procesos constructivos y de desarrollo para la institución.

- Fomenta la corresponsabilidad en el análisis y la búsqueda de soluciones a los conflictos. 
En suma, la mediación requiere que las partes se encuentren en un estado psicológico - cognitivo y emocional- que predisponga a la justicia relacional, un estado psicológico que pueden tenerlo ya desde el inicio de la mediación o ser el resultado de la actividad conjunta con el/la mediador/a y del propio proceso de relación interpersonal.

Pero existen barreras psicológicas y psicosociales a la mediación identificadas tanto en la investigación experimental realizada en el ámbito de la Psicología Social como a partir de la experiencia de mediadores/as y expertos/as en resolución de conflictos en la práctica (Deutsch y Coleman, 2012; Deutsch, Coleman y Marcus, 2006).

Los resultados de las investigaciones y las aplicaciones de la mediación en diferentes contextos han permitido identificar seis barreras psicológicas y psicosociales fundamentales a la mediación (Alcover, 2004; Bar-Tal y Halperin, 2011, 2013; Korobkin, 2006), las cuales se resumen a continuación: exceso de confianza optimista; sesgos atribucionales; efectos del contexto; devaluación reactiva; percepción de justicia procedimental y de la interacción; procesos y sesgos psicosociales.

\subsection{Exceso de confianza optimista}

La mayor parte de las personas tiende a mostrar un exceso de confianza acerca de los (buenos o deseados) resultados que va a obtener en eventos próximos, en lugar de los que realistamente son esperables. Esta percepción genera unas expectativas desproporcionadas acerca de los resultados que consideran esperables lograr en el proceso de mediación, de modo que se resisten a aceptar cualquier acuerdo que se aleje de lo esperado. También la mayoría de las personas tiende a creer que son mejores o más valiosas de lo que realmente son, y que merecen esos resultados excesivamente optimistas. Es una barrera en la mediación porque las personas anclan sus expectativas en esas creencias, de modo que esperan obtener más de lo que en la realidad suele plantearse como posible en el proceso.

\section{2. Sesgos atribucionales}

Los procesos de atribución consisten en buscar causas y explicaciones acerca del comportamiento propio (autoatribuciones) y de los demás (heteroatribuciones), procesos que pueden estar sesgados en función de las interpretaciones que las personas realizan acerca de las intenciones y propósitos de los otros. Las personas tienden a reaccionar de manera más tolerante ante los resultados negativos que obtienen cuando los atribuyen a causas inevitables o consecuencias de una situación, mientras que suelen reaccionar de modo muy negativo cuando atribuyen esos resultados nega- 
tivos a causas evitables, es decir, decisiones o actos negativos o perjudiciales que se atribuyen a los actos de otras personas. Los sesgos atribucionales se refieren a la tendencia a atribuir a causas personales e internas (es decir, provocadas por las intenciones y las decisiones deliberadas de los otros) las consecuencias negativas que experimentan en una relación o interacción interpersonal, sin tener en cuenta la influencia de factores situacionales o fuera del control de la persona. En general, producen enfado, enojo o ira hacia la otra parte, incluso cuando no hay razones objetivas para comprobar la causalidad del comportamiento atribuido.

\section{3. Efectos del contexto}

Las personas tienden a valorar las alternativas y a tomar una decisión según el contexto en que se produce, no por razones siempre objetivas o racionales. Es decir, la valoración de las opciones posibles o de las soluciones planteadas depende del contexto en que se producen y de las percepciones subjetivas de las partes, de modo que resultan difíciles de valorar de manera independiente de la situación. Uno de los efectos más consistentes es la tendencia a preferir una opción o una alternativa cuando la elección se enmarca como una entre varias ganancias que cuando esa misma elección se enmarca entre varias pérdidas. La valoración no se realiza en función de parámetros o criterios objetivos, sino del contexto en que se perciben. La investigación psicosocial ha mostrado evidencia consistente en apoyo del mayor efecto psicológico y emocional negativo que producen las pérdidas en las personas, que suelen ser mayor que el efecto positivo producido por la percepción de una ganancia. De aquí se deduce que cuando una posible opción o solución a un conflicto se percibe por una o por ambas partes como una pérdida -aunque sea una ligera pérdida-, la resistencia a aceptarla es mucho mayor, y que cuando la alternativa o el posible acuerdo supone una ganancia -más aún cuando esta es pequeña-, tiende a valorarse de manera ligeramente positiva, pero proporcionalmente tiene menor importancia que cuando se compara con el efecto negativo de una pequeña pérdida.

\section{4. Devaluación reactiva}

La reactancia es un fenómeno psicológico muy habitual en los seres humanos, y se refiere a una resistencia -no siempre consciente- no tanto a lo que se nos ofrece, se nos propone o se nos da, sino de quién procede la oferta o la propuesta, la cual se percibe como una amenaza a la libertad individual. Es especialmente sensible a la percepción de algún tipo de autoridad o de poder en la otra parte, hecho que incrementa la reactancia sin valorar el contenido real de la oferta o propuesta. La devaluación reactiva se refiere a 
la reacción que se produce ante una concesión que hace la otra parte, la cual se percibe menos deseable cuando realmente se ofrece de lo que se valoraba antes de que se ofreciera. Es decir, la concesión resulta menos valorada debido a su procedencia (la persona que la realiza), y no al valor en sí mismo de lo concedido.

\section{5. Percepción de justicia procedimental y de la interacción}

Las personas no solo valoran la justicia distributiva o del resultado -lo que obtienen tras lograr un acuerdo en un proceso de mediación-, sino también (y a veces, especialmente) la justicia del procedimiento que ha permitido alcanzar un resultado y la justicia de la interacción, o el trato recibido por la(s) otra(s) parte(s), incluido el/la mediador/a. Estos procesos psicosociales de percepción de justicia son muy relevantes, ya que influyen en la consideración de lo obtenido, con independencia de la valoración objetiva de los resultados.

\section{6. Procesos y sesgos psicosociales}

Percepciones acerca de las diferencias de valores, ideologías, visiones del mundo, posición social, naturaleza y características del otro. Esto genera la percepción de la otra parte en función de la categoría o grupo en el que el perceptor la incluye, y supone un proceso de despersonalización al percibir las características atribuidas al grupo o categoría en lugar de las características personales e idiosincrásicas de la otra parte. Esto tiene como resultado que tienden a mantenerse las percepciones y las diferencias intergrupales, en lugar de fomentar las percepciones y las relaciones interpersonales. Como consecuencia, se producen sesgos en la percepción de la otra parte, a quien suele atribuirse categorizaciones o estereotipos del grupo o categoría en el que se le incluye. Se mantienen los desacuerdos intergrupales por encima de los intereses y de los objetivos particulares.

\section{Conclusión}

La mediación es un proceso de resolución de conflictos basado en la comunicación y en la interacción entre las personas directamente implicadas y el/la mediador/a. En consecuencia, es preciso tener en cuenta la influencia de los procesos de percepción, atribución, cognitivos, emocionales y relacionales característicos de la naturaleza humana y de las relaciones interpersonales (Bar-Tal y Halperin, 2011, 2013). Como se ha tratado de mostrar sintéticamente en este artículo, para cualquier mediador/a es imprescindible conocer estos procesos y las barreras psicológicas y psicosociales que 
generan, de modo que este conocimiento les permita identificar cómo estos procesos pueden influir en el desarrollo y el desenlace de una mediación.

$\mathrm{El} / \mathrm{la}$ mediador/a debe intervenir si percibe que pueden estar influyendo en las percepciones de las partes, sesgando sus juicios, condicionando sus valoraciones y afectando a la posibilidad de llegar a un acuerdo. Aunque es recomendable que el/la mediador/a no intervenga en el contenido de la mediación (evitando realizar juicios o dar opiniones sobre las propuestas de las partes), sí ha de ser asertivo/a en cuanto al proceso, es decir, intervenir cuando considere que las partes no cumplen con las reglas de la mediación o cuando perciba claramente la influencia negativa de las barreras psicológicas y psicosociales mencionadas (Alcover, 2006).

No obstante, no puede olvidarse que los procesos psicológicos -cognitivos y emocionales- y psicosociales -percepción social, atribución, categorización, estereotipia, prejuicios - son en diverso grado ineludibles, y que de alguna manera van a influir en un proceso que nunca es completamente racional. Como señalaban Bar-Tal y Halperin (2013), la base de las barreras psicosociales internas consisten en creencias ideológicas que las personas han asumido a través de las influencias sociales, los estereotipos y los prejuicios, las cuales condicionan sus percepciones de los otros, de las causas de los conflictos y de los posibles acuerdos, y constituyen poderosos obstáculos para la resolución pacífica, negociada o mediada, de los conflictos. Superar estas barreras no es imposible, pero requiere de intensos procesos de auto-persuasión de todas las personas implicadas, así como de la influencia de los líderes y agentes sociales, en este caso concreto, de los miembros de la comunidad universitaria con mayor capacidad de influencia, para removerlos y modificar las percepciones acerca de los conflictos y de las otras partes de modo que sea posible valorar los beneficios de la comunicación, el diálogo, la mediación y la cultura de paz. La mediación es un proceso humano, relacional y contextual, y en consecuencia, sometido a las influencias de su naturaleza. Conocer estos procesos para su posible prevención o detección una vez que se han manifestado es una responsabilidad ineludible en la formación y la práctica de todo mediador/a.

\section{Bibliografía citada}

Alcover, C. M. (2004). "La atribución de responsabilidad". En I. Hoyo Sierra (coord.), Introducción a la Psicosociología del Derecho. Madrid: Dykinson, pp. 77-104.

Alcover, C. M. (2006). "La mediación como estrategia para la resolución de conflictos: una perspectiva psicosocial". En M. Gonzalo Quiroga (dir.) (2006), Métodos alternativos de solución de conflictos: perspectiva multidisciplinar. Madrid: Dykinson-Servicio de Publicaciones de la URJC, pp. 113-129. 
Alcover, C. M. (2009). "Ombudsing in Higher Education: A contingent model for mediation in university dispute resolution processes", The Spanish Journal of Psychology, 12, pp. 275-287.

Alcover, C. M. (2010). "Alternativas de mediación en contextos universitarios: una propuesta de modelo contingente y sus aplicaciones en la resolución de conflictos a través de la figura del defensor universitario". En N. González-Cuellar, A. Sanz y J. C. Ortiz (coords.), Mediación: un método de ? conflictos. Estudio interdisciplinar. Madrid: Colex, pp. 267-288.

Alcover, C. M. (2011). "Entrenamiento en mediación: las mejores prácticas para formar mediadores eficaces". En R. López San Luis (ed.), Aportaciones de la mediación en el marco de la prevención, gestión y solución de conflictos familiares. Granada: Comares, pp. 33-46.

Alcover, C. M. (2016). "Corresponsabilidad, reconciliación y mediación en la resolución de conflictos en contextos universitarios", Rued@, Revista Universidad, Ética y Derechos, 1, pp. 30-42.

BAR-TAL, D. y HALPERin, E. (2011). "Socio-psychological barriers to conflict resolution". En D., Bar-Tal (ed.), Intergroup conflicts and their resolution: A social psychological perspective. Nueva York: Psychology Press, pp. 217-240.

BAR-TAL, D. y HALPERIN, E. (2013). The nature of socio-psychological barriers to peaceful conflict resolution and ways to overcome them. Conflict \& Communication online, Vol. 12, No. 2, 2013. Disponible online: www.cco.regener-online.de (último acceso 1 de diciembre de 2017).

Bush, R. A. B. y Folger, J. P. (1994). The Promise of Mediation: Responding through Empowerment and Recognition. San Francisco, CA: Jossey Bass.

Casanovas, P. y Poblet, M. (2008). "Concepts and Fields of Relational Justice". En P. Casanovas et al. (eds.), Computable Models of the Law, LNAI 4884. Berlín: Springer, pp. 323-339.

Deutsch, M. y Coleman, P. T. (2012). Psychological Components of Sustainable Peace. Nueva York, NY: Springer.

Deutsch, M., Coleman, P. T., y Marcus, E. C. (2006). The Handbook of Conflict Resolution: Theory and Practice ( ${ }^{\mathrm{a}}$ ed.). San Francisco, CA: Jossey-Bass.

Edmondson, A. (1999). "Psychological safety and learning behavior in work teams", Administrative Science Quarterly, 44, pp. 350-383. 
Korobкin, R. (2006). Psychological impediments to mediation success: Theory and practice. Ohio State Journal on Dispute Resolution, 21, pp. 281-327.

Kressel, K. y Pruitt, D.G. (1989). Mediation Research. San Francisco, CA: Jossey Bass.

Moore, C. W. (2014). The Mediation Process: Practical Strategies for Resolving Conflict ( $\left.4^{\mathrm{a}} \mathrm{ed}.\right)$. San Francisco, CA: Jossey Bass.

PoITRAs, J. (2009). "Mediation: Depolarizing responsibilities to facilitate reconciliation", International Journal of Conflict Management, 21, pp. 4-19. 